\title{
Magnetic attitude control for satellites
}

\author{
Jan Tommy Gravdahl, \\ Department of Engineering Cybernetics \\ Norwegian University of Science and Technology \\ N-7491 TRONDHEIM, Norway
}

\begin{abstract}
In this paper it is proven that three-axis stabilization of satellites using magnetorqers is uniformly globally asymptotically stable (UGAS). The application of Matrosovs theorem allows for removal of the assumption that the geomagnetic (internal and external) field is periodic in order to establish this stability result. The results are applied to a model of a Norwegian pico-satellite.
\end{abstract}

\section{INTRODUCTION}

Active control of the attitude of a satellite can be achieved with a number of different actuators: reaction wheels, thrusters or magnetic coils. The development of a Norwegian pico-satellite required a control system that is both inexpensive and lightweight. This motivated the use of magnetic coils. As shown in [1], magnetic threeaxis stabilization of a spacecraft can be achieved by using feedback from magnetic field measurements and angular velocity. Asymptotic stability can then be established by assuming that the internal geomagnetic field (the component of the field having its cause in the iron core of the earth), as seen from the spacecraft, is periodic and then applying the Krasovskii-LaSalle theorem. However, the magnetometer on board the satellite will not only measure the internal geomagnetic field, but also the external magnetic field and a disturbance field induced by the spacecraft electronics, thus prohibiting the assumption of a periodic field. In this paper periodicity of the magnetic field will not be assumed, and UGAS will be proven for this time-varying system by using Matrosov's theorem.

\section{The NCUBE SATEllite}

On mission from The Norwegian Space Center and Andøya Rocket Range, four Norwegian universities and educational institutions have since 2001 participated in a program to develop a pico satellite. The satellite, nCube, is being designed and built by MSc-students, in accordance with the Cubesat concept: Mass is restricted to $1 \mathrm{~kg}$ and size is restricted to a cube measuring $0.1 \mathrm{~m}$ on all sides. A prototype of nCube is shown in Figure 1. The four partners are Narvik University College, Norwegian University of Science and Technology (NTNU), Agricultural University of Norway, and University of Oslo. The Attitude Determination and Control System (ADCS) is the responsibility of the Department of Engineering Cybernetics at NTNU.

The main mission of the satellite is to demonstrate ship traffic surveillance from a LEO satellite using the maritime Automatic Identification System (AIS) recently introduced

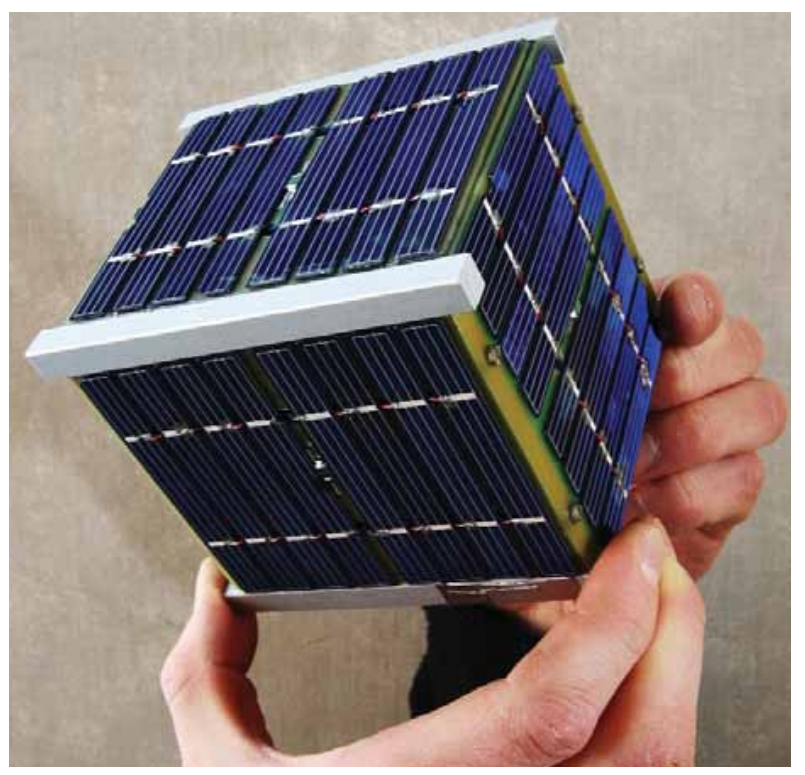

Fig. 1. Prototype of nCube illustrating the size and solar panels.

by the International Maritime Organization (IMO). The AIS system is based on VHF transponders located on board ships. These transponders broadcast the position, speed, heading and other relevant information from the ships at regular time intervals. The main objective of the satellite is to receive, store and retransmit at least one AIS-message from a ship. Another objective of the satellite project is to demonstrate reindeer herd monitoring from space by equipping a reindeer with an AIS transponder during a limited experimental period. In addition, the satellite should maintain communications and digipeater operations using amateur frequencies. A third objective is to demonstrate efficient attitude control using passive gravity gradient stabilization and active magnetic torquers.

The satellite will be placed in a low earth sun synchronous orbit with a perigee of approximately $700 \mathrm{~km}$, and as circular as possible. The inclination will be close to $98^{\circ}$. The launch is scheduled to early 2005. For further information on the nCube project and detailed description of the satellite the reader is referred to [2] or [3]

The attitude and angular velocity of the spacecraft will be determined by integrating measurements of the magnetic field and measurements of the sun vector in an extended Kalman filter. The magnetic field will be measured using a 


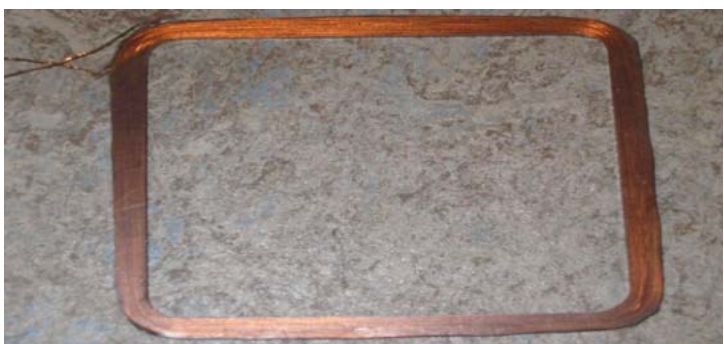

Fig. 2. Photo of one of the torque coils

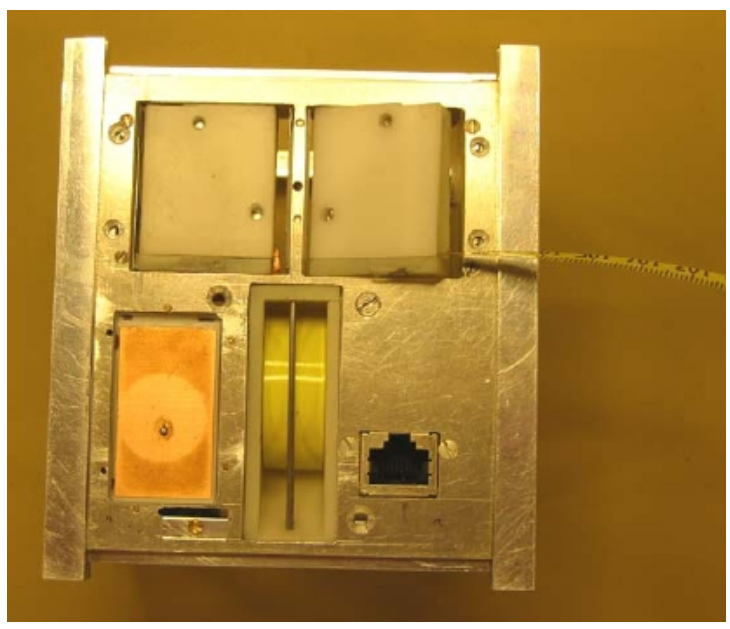

Fig. 3. Photo of the nadir surface of Ncube. The gravity boom is coiled up and restrained inside a box.

digital magnetometer and the sun vector will be measured by monitoring the current output from the solar panels which cover all the sides of the cube. For actuation of the satellite, magnetic torque coils will be used. A prototype coil is shown in Figure 2. A gravity boom is constructed from measuring tape which will act as the boom rod. A tip-mass made of lead is attached to the boom. The coiled up boom is shown in the lower middle in Figure 3. For details on sensors and actuators and the construction of the satellite in general, see [4].

\section{Modeling}

\section{A. Satellite model}

1) Coordinate frames and kinematics: Earth-Centered Inertial (ECI) Reference Frame The origin of this frame is located in the center of the earth. This reference frame will be denoted $i$, and the earth rotates around its $z$-axis. The $x$-axis points towards the vernal equinox.

Orbit Reference Frame The orbit frame origin coincides with the spacecraft center. The origin rotate at an angular velocity $\omega_{o}$ relative to the ECI frame and has its $z$-axis pointed towards the center of the earth. The $x$-axis points in the spacecraft's direction of motion tangentially to the orbit. The satellite attitude is described by roll, pitch and yaw which is the rotation around the $x-, y$-, and $z$-axis respectively. The orbit reference frame is denoted $o$.

Body Reference Frame The body frame shares it's origin with the orbit frame and is denoted $b$. The rotation between the orbit frame and the body frame is used to represent the spacecraft's attitude. It's axes are locally defined in the spacecraft, with the origin in the center of gravity or the center of the volume. The nadir side of the spacecraft, intended to point towards the earth, is in the $z$-axis direction.

The rotation matrix $\mathbf{R}$ from frame $a$ to frame $b$ is denoted $\mathbf{R}_{a}^{b}$. Rotation matrices are members of the special orthogonal group of order three:

$$
\mathbf{R} \in S O(3)=\left\{\mathbf{R} \mid \mathbf{R} \in \mathbb{R}^{3 \times 3}, \mathbf{R}^{T} \mathbf{R}=\mathbf{I}, \operatorname{det} \mathbf{R}=1\right\}
$$

where $\mathbf{I}$ is the $3 \times 3$ identity matrix. A transformation of a vector $\mathbf{r}$ from frame $a$ to frame $b$ is written $\mathbf{r}^{b}=\mathbf{R}_{a}^{b} \mathbf{r}^{a}$. From the orthogonal property in (1), it can be shown [5] that the time derivative of $\mathbf{R}_{a}^{b}$ can be written as

$$
\dot{\mathbf{R}}_{a}^{b}=\mathbf{S}\left(\boldsymbol{\omega}_{a b}^{a}\right) \mathbf{R}_{a}^{b}=\mathbf{R}_{a}^{b} \mathbf{S}\left(\boldsymbol{\omega}_{a b}^{b}\right)
$$

where $\boldsymbol{\omega}_{a b}^{b}$ is the angular velocity of frame $b$ relative to frame $a$ represented in frame $b$, and $\mathbf{S}(\boldsymbol{\omega})=\boldsymbol{\omega} \times$ is the cross product operator. Generally, the matrix $\mathbf{R}_{o}^{b}$ can be written as

$$
\mathbf{R}_{o}^{b}=\left(\mathbf{c}_{1}^{b} \mathbf{c}_{2}^{b} \mathbf{c}_{3}^{b}\right)
$$

where $\mathbf{c}_{1}^{b}=\left(c_{i x}^{b} c_{i y}^{b} c_{i z}^{b}\right)^{T}$ are column vectors. The $c_{3}^{b}$ vector is the projection of the $z_{0}$-axis in the body frame. If $\mathbf{c}_{3}^{b}=\left(\begin{array}{lll}0 & 0 & 1\end{array}\right)^{T}$, the $z_{b}$-axis is aligned with the $z_{0}$-axis. In this paper $c_{i z}^{b}$ will be frequently used as a measurement of deviation between the $z_{b}$-axis and the $z_{o}$-axis. From (2) and (3), it follows that

$$
\dot{\mathbf{c}}_{i}^{b}=\mathbf{S}\left(\mathbf{c}_{i}^{b}\right) \boldsymbol{\omega}_{o b}^{b} .
$$

2) Dynamics: Using Euler's moment equation, the attitude dynamics of the satellite can be derived as

$$
\mathbf{J} \dot{\boldsymbol{\omega}}_{i b}^{b}+\boldsymbol{\omega}_{i b}^{b} \times\left(\mathbf{J} \boldsymbol{\omega}_{i b}^{b}\right)=\boldsymbol{\tau}^{b},
$$

where $\mathbf{J}$ is the inertia matrix of the satellite, $\boldsymbol{\omega}_{i b}^{b}$ is the angular velocity of the $b$-frame with respect to the $i$-frame, decomposed in the $b$-frame, and

$$
\boldsymbol{\tau}^{b}=\boldsymbol{\tau}_{m}^{b}+\boldsymbol{\tau}_{g}+\boldsymbol{\tau}_{a}+\boldsymbol{\tau}_{s}+\boldsymbol{\tau}_{m}
$$

is the torque acting on the satellite. The torque $\tau_{m}^{b}$ generated by the magnetorquers can be modelled as

$$
\boldsymbol{\tau}_{m}^{b}=\mathbf{m}^{b} \times \mathbf{B}^{b},
$$

where $\mathbf{m}^{b}$ is the magnetic dipole moment generated by the coils and $\mathbf{B}^{b}=\left(B_{x} B_{y} B_{z}\right)^{T}$ is the local geomagnetic field vector. The magnetic dipole moment is given by

$$
\mathbf{m}^{b}=\mathbf{m}_{x}^{b}+\mathbf{m}_{y}^{b}+\mathbf{m}_{z}^{b}=\left(\begin{array}{c}
N_{x} i_{x} A_{x} \\
N_{y} i_{y} A_{y} \\
N_{z} i_{z} A_{z}
\end{array}\right)=\left(\begin{array}{c}
m_{x} \\
m_{y} \\
m_{z}
\end{array}\right),
$$




\begin{tabular}{ll} 
Disturbance & Magnitude $[\mathrm{Nm}]$ \\
\hline \hline Gravity gradient & $1.0382 \cdot 10^{-7}$ \\
Aerodynamic drag & $3.65 \cdot 10^{-9}$ \\
Solar radiation & $1.5371 \cdot 10^{-8}$ \\
Internal electronics field & $1 \cdot 10^{-6}$ \\
\multicolumn{2}{l}{} \\
\hline
\end{tabular}

TABLE I

DISTURBANCE TORQUES FOR NCUBE

where $N_{k}$ is the number of windings in the magnetic coil on the axis in the $k$-direction, $i_{k}$ is the current in the coil and $A_{k}$ is the coil area. The disturbance torques $\tau_{g}, \boldsymbol{\tau}_{a}, \boldsymbol{\tau}_{s}$ and $\boldsymbol{\tau}_{m}$ will be defined later.

The angular velocity of the body frame with respect to the orbit frame can be found by

$$
\boldsymbol{\omega}_{o b}^{b}=\boldsymbol{\omega}_{i b}^{b}-\mathbf{R}_{o}^{b} \boldsymbol{\omega}_{i o}^{o}=\boldsymbol{\omega}_{i b}^{b}-\omega_{o} \mathbf{c}_{1}^{b} .
$$

\section{B. Disturbance torques}

A satellite is subject to small but persistent disturbance torques. Unless actively resisted the disturbances will reorient the satellite. The main disturbances are briefly discussed below. The discussion is based on [6] and [7].

a) Gravity gradient torque: The gravity gradient torque $\tau_{g}$, written in the $b$-frame as

$$
\boldsymbol{\tau}_{g}^{b}=3 \omega_{0}^{2} \mathbf{c}_{3}^{b} \times\left(\mathbf{J} \mathbf{c}_{3}^{b}\right),
$$

where $\omega_{0} \approx\left(\mu / R_{0}^{3}\right)^{1 / 2}, \mu=G M$ is the Earth's gravitational coefficient and $R_{0}$ is the distance to the Earth's center, will affect a non symmetric body in the Earth's gravity field. This effect can be exploited, and will be in the case of nCube, with a gravity boom for passive stabilization.

b) Magnetic fields: The electronics in the satellite may create an unwanted residual magnetic dipole. This field will interact with the Earth's geomagnetic field. The resulting torque can be written as

$$
\tau_{m}=D B,
$$

where $D$ is the residual dipole of the satellite and $B$ is the Earth's magnetic field.

Also, disturbances may have their source in the external geomagnetic field. This field varies on a faster time-scale than the internal geomagnetic field. Moreover, the external field may very in an unpredictable manner.

c) Other: Other disturbances that are taken into account for the nCube satellite, but not discussed in detail here, include aerodynamic torque, $\tau_{a}$, solar radiation torque, $\boldsymbol{\tau}_{s}$, and boom distortion. In Table I, the worst case numerical values for nCube are presented.

\section{Controller Design}

\section{A. Energy considerations}

An important tool in control theory is the use of energybased controllers based on Lyapunov designs and passivity [5]. In this section expressions for the satellite's energy is presented, and a suitable Lyapunov function candidate and its derivative is found.

The energy of the satellite can be divided into kinetic and potential energy. The kinetic energy is mainly due to rotation in the inertial and orbit frame, while the most important source to potential energy is the gravity gradient and gyro effects due to revolution about the Earth. The expressions for kinetic and potential energy is based on [8], [9] and [10]. From a control perspective the rotation of the body frame with respect to the orbit frame is most interesting. Assuming a near circular orbit, and therefore a constant orbital rate $\omega_{o}$, the kinetic energy can be written

$$
E_{k i n}=\frac{1}{2}\left(\boldsymbol{\omega}_{o b}^{b}\right)^{T} \mathbf{J} \boldsymbol{\omega}_{o b}^{b} .
$$

The potential energy due to the gravity gradient is

$$
E_{g g}=\frac{3}{2}\left(\omega_{o}^{2}\left(\mathbf{c}_{3}^{b}\right)^{T} \mathbf{J} \mathbf{c}_{3}^{b}-I_{z}\right),
$$

and the potential energy due to the revolution of the satellite about the Earth is given by

$$
E_{\text {gyro }}=\frac{1}{2} \omega_{o}^{2}\left(I_{x}-\left(\mathbf{c}_{1}^{b}\right)^{T} \mathbf{J c}_{1}^{b}\right) .
$$

Defining

$$
\mathbf{x}=\left(\begin{array}{lllll}
\boldsymbol{\omega}_{o b}^{b} & c_{21} & c_{31} & c_{13} & c_{23}
\end{array}\right)^{T} \in \mathbb{R}^{7},
$$

and using (8), (9) and (10), it can be seen that the energy function $V$ defined by

$$
\begin{aligned}
V(\mathbf{x})= & E_{k i n}+E_{g g}+E_{\text {gyro }}=\frac{1}{2}\left(\boldsymbol{\omega}_{o b}^{b}\right)^{T} \mathbf{J} \boldsymbol{\omega}_{o b}^{b} \\
& +\frac{3}{2} \omega_{o}^{2}\left(\left(I_{x}-I_{z}\right) c_{13}^{2}+\left(I_{y}-I_{z}\right) c_{23}^{2}\right) \\
& +\frac{1}{2} \omega_{o}^{2}\left(\left(I_{x}-I_{y}\right) c_{21}^{2}+\left(I_{x}-I_{z}\right) c_{31}^{2}\right)
\end{aligned}
$$

satisfies $V(\mathbf{0})=0$. The simplifications in the last two terms of (11) follows from the fact that $\mathbf{R}_{o}^{b}$ is orthogonal. In order to ensure that $V$ is positive definite, that is $V>0 \forall \mathbf{x} \neq \mathbf{0}$, we require that $I_{x}>I_{y}>I_{z}$. For use in the stability analysis of the controller we need an expression for the time derivative of (11)

$$
\dot{V}=\left(\boldsymbol{\omega}_{o b}^{b}\right)^{T} \mathbf{J} \dot{\boldsymbol{\omega}}_{o b}^{b}+3 \omega_{o}^{2}\left(\mathbf{c}_{3}^{b}\right)^{T} \mathbf{J} \dot{\mathbf{c}}_{3}^{b}-\omega_{o}^{2}\left(\mathbf{c}_{1}^{b}\right)^{T} \mathbf{J} \dot{\mathbf{c}}_{1}^{b} .
$$

It follows from (5) and (7) that the satellite dynamics, considering the gravity gradient and magnetic coil torques only, can be written as

$$
\mathbf{J} \dot{\boldsymbol{\omega}}_{i b}^{b}+\boldsymbol{\omega}_{i b}^{b} \times\left(\mathbf{J} \boldsymbol{\omega}_{i b}^{b}\right)=3 \omega_{0}^{2} \mathbf{c}_{3}^{b} \times\left(\mathbf{J c}_{3}^{b}\right)+\boldsymbol{\tau}_{m}^{b} .
$$

Using (4), (13) and the relations $\boldsymbol{\omega}_{i b}=\boldsymbol{\omega}_{o b}+\omega_{0} \mathbf{c}_{1}^{b}$ and $\left(\boldsymbol{\omega}_{o b}^{b}\right)^{T} \mathbf{S}\left(\boldsymbol{\omega}_{o b}^{b}\right)=\mathbf{0},(12)$ is written

$$
\begin{aligned}
\dot{V}= & \left(\boldsymbol{\omega}_{o b}^{b}\right)^{T}\left(3 \omega_{0}^{2} \mathbf{S}\left(\mathbf{c}_{3}^{b}\right) \mathbf{J} \mathbf{c}_{3}^{b}+\boldsymbol{\tau}_{m}^{b}-\omega_{0} \mathbf{J S}\left(\mathbf{c}_{1}^{b}\right) \boldsymbol{\omega}_{o b}^{b}\right. \\
& \left.-\omega_{0} \mathbf{S}\left(\mathbf{c}_{1}^{b}\right) \mathbf{J} \boldsymbol{\omega}_{o b}^{b}-\omega_{0}^{2} \mathbf{S}\left(\mathbf{c}_{1}^{b}\right) \mathbf{J} \mathbf{c}_{1}^{b}\right) \\
& +3 \omega_{o}^{2}\left(\mathbf{c}_{3}^{b}\right)^{T} \mathbf{J S}\left(\mathbf{c}_{3}^{b}\right) \boldsymbol{\omega}_{o b}^{b}-\omega_{o}^{2}\left(\mathbf{c}_{1}^{b}\right)^{T} \mathbf{J S}\left(\mathbf{c}_{1}^{b}\right) \boldsymbol{\omega}_{o b}^{b}
\end{aligned}
$$


Since $\mathbf{S}^{T}(\mathbf{x})=-\mathbf{S}(\mathbf{x}),(14)$ is reduced to

$$
\dot{V}=\left(\boldsymbol{\omega}_{o b}^{b}\right)^{T} \boldsymbol{\tau}_{m}^{b} .
$$

Remark 1: The equilibrium given by $\mathrm{x}=$ $\left(\begin{array}{lllll}\boldsymbol{\omega}_{o b}^{b} & c_{21} & c_{31} & c_{13} & c_{23}\end{array}\right)^{T}=\mathbf{0}$, corresponds to four equilibria

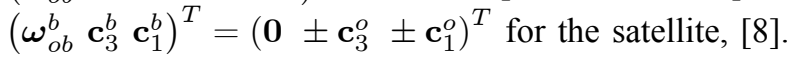

\section{B. Detumbling}

When the satellite is released from the launcher it will have an initial angular velocity. Before the boom can be deployed, angular velocity must be reduced and the body frame must be aligned with the orbit frame. During detumbling the kinetic energy of the satellite is dumped and the angular velocity of the body frame with respect to the inertial frame is to be reduced to a value below $\boldsymbol{\omega}_{o b}^{b}<\left(5 \cdot 10^{-3} 5 \cdot 10^{-3} 5 \cdot 10^{-3}\right)^{T}$. The only sensor available in this mode will be the magnetometer. After the rate detumbling phase the satellite may have an arbitrary attitude. Before the boom can be deployed we must ensure that the body $z_{b}$-axis is aligned with the orbit $z_{o}$-axis. If the boom is deployed in the opposite direction it may be difficult to turn the satellite. For boom deployment we require that the deviation between the $z_{b}$ and $z_{o}$ axes is less than $30^{\circ}$.

The objective of the rate detumbling controller is to dissipate the kinetic energy of the satellite. A controller which uses only rate measurements from the magnetometer is suggested below. The controller is proposed in [10], [11] and [9].

Proposition 1: The control law

$$
\mathbf{m}^{b}=-k \dot{\mathbf{B}}^{b}-\mathbf{m}_{c},
$$

where $\mathbf{m}_{c}=\left(\begin{array}{lll}0 & 0 & m_{c}\end{array}\right)^{T}$ will dissipate the kinetic energy of the satellite and align it with the local geomagnetic field.

Proof: To prove that the energy is dissipated, Lyapunov theory will be used. The proof is based on [10] and [11]. Combining (6) and (16), the control torque $\boldsymbol{\tau}_{m}^{b}$ gives

$$
\boldsymbol{\tau}_{m}^{b}=\mathbf{m}^{b} \times \mathbf{B}^{b}=\left(-k \dot{\mathbf{B}}^{b}-\mathbf{m}_{c}\right) \times \mathbf{B}^{b} .
$$

We note that the magnetic field vector $\mathbf{B}^{b}$ can be written as $\mathbf{B}^{b}=\mathbf{R}_{i}^{b} \mathbf{B}^{i}$, and consequently

$$
\dot{\mathbf{B}}^{b}=\dot{\mathbf{R}}_{i}^{b} \mathbf{B}^{i}+\mathbf{R}_{i}^{b} \dot{\mathbf{B}}^{i}=\mathbf{B}^{b} \times \boldsymbol{\omega}_{i b}^{b}+\mathbf{R}_{i}^{b} \dot{\mathbf{B}}^{i} .
$$

Near the North and South Poles, $\mathbf{B}^{b}$ is approximately constant. Equation (18) can therefore be approximated as $\dot{\mathbf{B}}^{b} \approx \mathbf{B}^{b} \times \boldsymbol{\omega}_{i b}^{b}$. This assumption is valid only in the polar regions. When the boom is stowed, the gravity gradient will be very small and can be neglected, however the constant term in (16) will contribute to the potential energy. Thus, the sum of kinetic $T$ and potential energy $U$ is

$$
V=T+U=\frac{1}{2}\left(\boldsymbol{\omega}_{i b}^{b}\right)^{T} \mathbf{J} \boldsymbol{\omega}_{i b}^{b}+\left|\mathbf{m}_{c}\right|\left|\mathbf{B}^{b}\right|+\mathbf{m}_{c}^{T} \mathbf{B}^{b} .
$$

Assuming a constant magnitude of the geomagnetic field in the polar regions, the time derivative of (19) is

$$
\dot{V}=\left(\boldsymbol{\omega}_{i b}^{b}\right)^{T} \boldsymbol{\tau}_{m}^{b}+\mathbf{m}_{c}^{T} \dot{\mathbf{B}}^{b},
$$

and using (17) it follows that

$$
\begin{aligned}
\dot{V} & =\left(\boldsymbol{\omega}_{i b}^{b}\right)^{T}\left(\left(-k \dot{\mathbf{B}}^{b}-\mathbf{m}_{c}\right) \times \mathbf{B}^{b}\right)+\mathbf{m}_{c}^{T} \dot{\mathbf{B}}^{b} \\
& =-k\left(\dot{\mathbf{B}}^{b}\right)^{T} \dot{\mathbf{B}}^{b}
\end{aligned}
$$

which is negative semidefinite. We conclude that energy is dissipated and the angular velocities are reduced.

Remark 2: The $z_{b}$-axis will tend to point along $\mathbf{B}^{i}$. This is not shown in the analysis above, but a proof can be found in [9] or [11]. Near the poles $\mathbf{B}^{i}$ points vertically upwards, meaning that in the polar regions the deviation of the $z_{b}$ axis from nadir will be relatively small. This can be utilized for boom deployment.

\section{Stabilization}

We will here use the same control law as in [10] and [1] with the correction suggested by [12].. Asymptotic stability of Wisniewski's controller was proven by assuming Earth's magnetic field to be periodic and then using the Krasovskii-LaSalle theorem [13]. As discussed in Section III-B.0.b, the magnetic field may vary with time in an unpredictable manner. Our contribution to previous work is to use Matrosov's theorem [14] as stated in [15] in order to prove uniform global asymptotic stability (UGAS) of the equilibrium without assuming periodicity of the geomagnetic field $\mathbf{B}^{b}(t)$.

Proposition 2: The control law

$$
\mathbf{m}^{b}=\mathbf{H}\left(\boldsymbol{\omega}_{o b}^{b} \times \mathbf{B}^{b}\right),
$$

makes the origin, $\mathbf{x}=\mathbf{0}$ of the system (13), (4) GUAS.

Proof: Define

$$
\begin{aligned}
V_{1}= & V=\frac{1}{2}\left(\boldsymbol{\omega}_{o b}^{b}\right)^{T} \mathbf{J} \boldsymbol{\omega}_{o b}^{b}+\frac{3}{2}\left(\omega_{o}^{2}\left(\mathbf{c}_{3}^{b}\right)^{T} \mathbf{J} \mathbf{c}_{3}^{b}-I_{z}\right) \\
& +\frac{1}{2} \omega_{o}^{2}\left(I_{x}-\left(\mathbf{c}_{1}^{b}\right)^{T} \mathbf{J} \mathbf{c}_{1}^{b}\right),
\end{aligned}
$$

where the LFK in equation (11) has been used. Using (20) and calculating the time derivative of (21) along the trajectories of (13), (4) results in

$$
\begin{aligned}
\dot{V}_{1} & =\left(\boldsymbol{\omega}_{o b}^{b}\right)^{T} \mathbf{H}\left(\boldsymbol{\omega}_{o b}^{b} \times \mathbf{B}^{b}\right) \times \mathbf{B}^{b} \\
& =-\left(\boldsymbol{\omega}_{o b}^{b}\right)^{T} \mathbf{S}^{T}\left(\mathbf{B}^{b}\right) \mathbf{H} \mathbf{S}\left(\mathbf{B}^{b}\right) \boldsymbol{\omega}_{o b}^{b}=Y_{1} \leq 0,
\end{aligned}
$$

as shown in equations (12) to (15). The Lyapunov function candidate (21) is positive definite and its time derivative (22) is negative semidefinite. It follows that the origin is UGS, and Assumption 1 of Theorem 1 in [15] is satisfied. Moreover, Assumption 2 is satisfied for $i=1$. Define the auxiliary function $V_{2}$.

$$
V_{2}=-\mathbf{c}_{3} \mathbf{J}^{T} \mathbf{S}^{T}\left(\mathbf{c}_{3}\right) \mathbf{J} \boldsymbol{\omega}_{o b}^{b},
$$

where the $b$ superscript on $\mathbf{c}_{1,2}^{b}$ has been dropped for notational convenience. Now,

$$
\begin{aligned}
\dot{V}_{2}= & -\dot{\mathbf{c}}_{3} \mathbf{J}^{T} \mathbf{S}^{T}\left(\mathbf{c}_{3}\right) \mathbf{J} \boldsymbol{\omega}_{o b}^{b}-\mathbf{c}_{3} \mathbf{J}^{T} \mathbf{S}^{T}\left(\dot{\mathbf{c}}_{3}\right) \mathbf{J} \boldsymbol{\omega}_{o b}^{b} \\
& -\mathbf{c}_{3} \mathbf{J}^{T} \mathbf{S}^{T}\left(\mathbf{c}_{3}\right) \mathbf{J} \dot{\boldsymbol{\omega}}_{o b}^{b} .
\end{aligned}
$$


As the system is stable, it follows that the states are bounded. Using the same notation as in [15], we let the number $\nu$ denote a generic bound on continuous functions, and $\dot{V}_{2}$ can be upper bounded as

$$
\dot{V}_{2} \leq-\mathbf{c}_{3} \mathbf{J}^{T} \mathbf{S}^{T}\left(\mathbf{c}_{3}\right) \mathbf{J} \dot{\boldsymbol{\omega}}_{o b}^{b}+\nu_{1}\left|\boldsymbol{\omega}_{o b}^{b}\right|,
$$

and using (13) further bounded as

$\dot{V}_{2} \leq-\mathbf{c}_{3}^{T} \mathbf{J}^{T} \mathbf{S}^{T}\left(\mathbf{c}_{3}\right)\left(3 \omega_{0}^{2} \mathbf{S}\left(\mathbf{c}_{3}\right) \mathbf{J} \mathbf{c}_{3}-\omega_{0}^{2} \mathbf{S}\left(\mathbf{c}_{1}\right) \mathbf{J} \mathbf{c}_{1}\right)+\nu_{2}\left|\boldsymbol{\omega}_{o b}^{b}\right|$

The second term in $\dot{V}_{2}$ satisfies

$$
\begin{aligned}
\omega_{0}^{2} \mathbf{c}_{3}^{T} \mathbf{J}^{T} \mathbf{S}^{T}\left(\mathbf{c}_{3}\right) \mathbf{S}\left(\mathbf{c}_{1}\right) \mathbf{J} \mathbf{c}_{1} & =-\omega_{0}^{2} \mathbf{c}_{3}^{T} \mathbf{J}^{T} \mathbf{S}\left(\mathbf{c}_{3}\right) \mathbf{S}\left(\mathbf{c}_{1}\right) \mathbf{J} \mathbf{c}_{1} \\
& =-\omega_{0}^{2} \mathbf{c}_{3}^{T} \mathbf{J}^{T}\left(\mathbf{c}_{1} \mathbf{c}_{3}^{T}-\mathbf{c}_{3}^{T} \mathbf{c}_{1} \mathbf{I}\right) \mathbf{J} \mathbf{c}_{1} \\
& =-\omega_{0}^{2} \mathbf{c}_{3}^{T} \mathbf{J}^{T} \mathbf{c}_{1} \mathbf{c}_{3}^{T} \mathbf{J} \mathbf{c}_{1} \\
& =-\omega_{0}^{2}\left(\mathbf{c}_{3}^{T} \mathbf{J}^{T} \mathbf{c}_{1}\right)^{2}<0
\end{aligned}
$$

so that

$$
\dot{V}_{2} \leq-3 \omega_{0}^{2} \mathbf{c}_{3}^{T} \mathbf{J}^{T} \mathbf{S}^{T}\left(\mathbf{c}_{3}\right) \mathbf{S}\left(\mathbf{c}_{3}\right) \mathbf{J} \mathbf{c}_{3}+\nu_{2}\left|\boldsymbol{\omega}_{o b}^{b}\right| .
$$

Now, we see that $Y_{1} \equiv 0$ implies

$$
\dot{V}_{2} \leq-2 \omega_{0}^{2} \mathbf{c}_{3} \mathbf{J}^{T} \mathbf{S}^{T}\left(\mathbf{c}_{3}\right) \mathbf{S}\left(\mathbf{c}_{3}\right) \mathbf{J} \mathbf{c}_{3}=Y_{2} \leq 0,
$$

and Assumption 2 and 3 are satisfied for $i=2$. Defining the auxiliary function $V_{3}$ as

$$
V_{3}=\mathbf{c}_{1} \mathbf{J}^{T} \mathbf{S}^{T}\left(\mathbf{c}_{1}\right) \mathbf{J} \boldsymbol{\omega}_{o b}^{b},
$$

and calculating its time derivative in the same manner as for $\dot{V}_{2}$ results in

$$
\begin{aligned}
\dot{V}_{3}= & \dot{\mathbf{c}}_{1} \mathbf{J}^{T} \mathbf{S}^{T}\left(\mathbf{c}_{1}\right) \mathbf{J} \boldsymbol{\omega}_{o b}^{b}+\mathbf{c}_{1} \mathbf{J}^{T} \mathbf{S}^{T}\left(\dot{\mathbf{c}}_{1}\right) \mathbf{J} \boldsymbol{\omega}_{o b}^{b} \\
& +\mathbf{c}_{1} \mathbf{J}^{T} \mathbf{S}^{T}\left(\mathbf{c}_{1}\right) \mathbf{J} \dot{\boldsymbol{\omega}}_{o b}^{b} \\
\leq & \mathbf{c}_{1} \mathbf{J}^{T} \mathbf{S}^{T}\left(\mathbf{c}_{1}\right) \mathbf{J} \dot{\omega}_{o b}^{b}+\nu_{3}\left|\boldsymbol{\omega}_{o b}^{b}\right| \\
\leq & \mathbf{c}_{1} \mathbf{J}^{T} \mathbf{S}^{T}\left(\mathbf{c}_{1}\right)\left(3 \omega_{0}^{2} \mathbf{S}\left(\mathbf{c}_{3}\right) \mathbf{J} \mathbf{c}_{3}\right. \\
& \left.-\omega_{0}^{2} \mathbf{S}\left(\mathbf{c}_{1}\right) \mathbf{J} \mathbf{c}_{1}\right)+\nu_{4}\left|\boldsymbol{\omega}_{o b}^{b}\right| \\
\leq & -\omega_{0}^{2} \mathbf{c}_{1} \mathbf{J}^{T} \mathbf{S}^{T}\left(\mathbf{c}_{1}\right) \mathbf{S}\left(\mathbf{c}_{1}\right) \mathbf{J} \mathbf{c}_{1} \\
& +\nu_{4}\left|\boldsymbol{\omega}_{o b}^{b}\right|+\nu_{5}\left|\mathbf{c}_{3}\right|
\end{aligned}
$$

where the same technique as for $\dot{V}_{2}$ has been used. Now $Y_{1} \equiv Y_{2} \equiv 0$ implies

$$
\dot{V}_{3} \leq-\omega_{0}^{2} \mathbf{c}_{1} \mathbf{J}^{T} \mathbf{S}^{T}\left(\mathbf{c}_{1}\right) \mathbf{S}\left(\mathbf{c}_{1}\right) \mathbf{J} \mathbf{c}_{1}=Y_{3} \leq 0,
$$

and Assumption 2 and 3 are satisfied for $i=3$. Finally $Y_{i}=0, i=\{1,2,3\} \Longrightarrow \mathbf{x}=\mathbf{0}$, and Assumption 4 of [15] is satisfied and the result follows.

Remark 3: In the two equilibria $\left(\boldsymbol{\omega}_{o b}^{b} \mathbf{c}_{3}^{b} \mathbf{c}_{1}^{b}\right)=$ $\left(\mathbf{0}-\mathbf{c}_{3}^{o} \pm \mathbf{c}_{1}^{o}\right)$ the boom is pointing in the wrong direction.

\section{Simulations}

Now the controllers for detumbling and stabilization will be simulated. The parameters of the model used in the simulations are: Body size $10 \times 10 \times 10 \mathrm{~cm}$, boom length: $1.5 \mathrm{~m}$, moments of inertia, boom stowed: $I_{x}=$ $0.0621 \mathrm{kgm}^{2}, I_{y}=0.0606 \mathrm{kgm}^{2}, I_{z}=0.0031 \mathrm{kgm}^{2}$, moments of inertia, boom deployed: $I_{x}=0.3210 \mathrm{kgm}^{2}, I_{y}=$
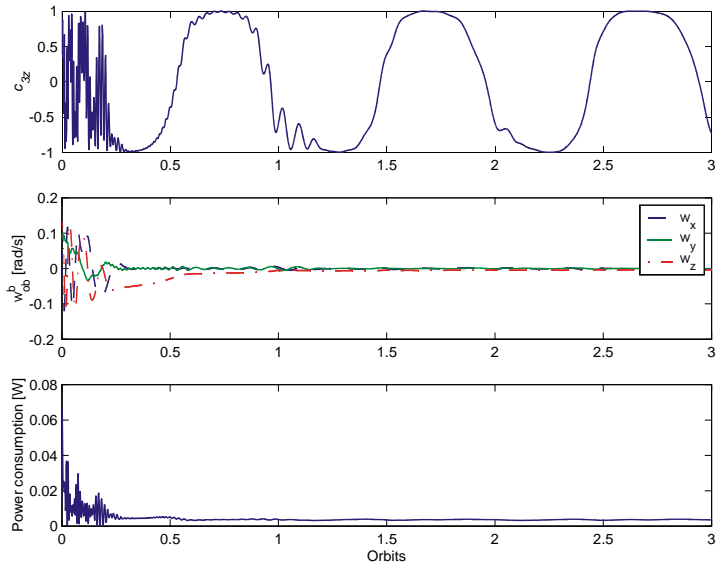

Fig. 4. Detumble mode simulation

$0.1806 \mathrm{kgm}^{2}, I_{z}=0.0031 \mathrm{kgm}^{2}$, maximum magnetic moment from the coils: $0.1 \mathrm{Am}^{2}$. Feedback from the magnetic field is done using the The International Geomagnetic Reference Field, IGRF. It is an approximation, near and above the Earth's surface, to that part of the Earth's magnetic field which has its origin in the earths core. The IGRF specifies the numerical coefficients of a truncated spherical harmonic series and together with an orbit estimator, an estimate of the magnetic field is made.

\section{A. Detumbling mode}

The detumbling mode controller (16) was simulated with initial values: $\omega_{i b}^{b}=\left(\begin{array}{lll}0.1 & 0.1 & 0.09\end{array}\right)^{T} \mathrm{rad} / \mathrm{s}$ and controller parameters: $k=10^{4}$ and $m_{c}=-0.01 \mathrm{Am}^{2}$. In Figure 4 it is shown that the angular velocities are quickly reduced and the $z_{b}$-axis aligns itself with the geomagnetic field vector.

\section{B. Stabilization mode}

In Figure 5, the Euler angles of the satellite using the stabilizing controller (20) is shown. A disturbance (white noise) with amplitude $\pm 10^{-5} \mathrm{~T}$ has been added to the magnetometer measurements, and as can bee seen the satellite is stabilized. The magnetic moments from the coils are shown in Figure 6. The total energy $V$ of the satellite as well as the angular velocity is shown in Figure 7.

\section{FURTHER WORK}

Ongoing work in the ADCS-part of this project includes final design of the determination system and implementation of the complete ADCS on microcontrollers. The final satellite will undergo assembly and testing during autumn 2004 and launch is planned for spring 2005 .

\section{CONCLUDING REMARKS}

It has been shown that the equilibrium of a time-varying system consisting of spacecraft attitude dynamics and a nonlinear control law using feedback from magnetic field measurements and angular velocity is UGAS. Magnetic 


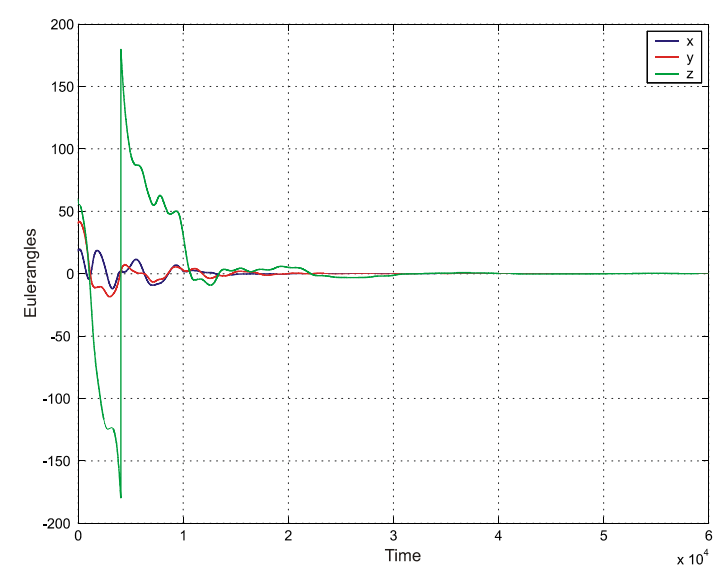

Fig. 5. The stabilized attitude of nCube

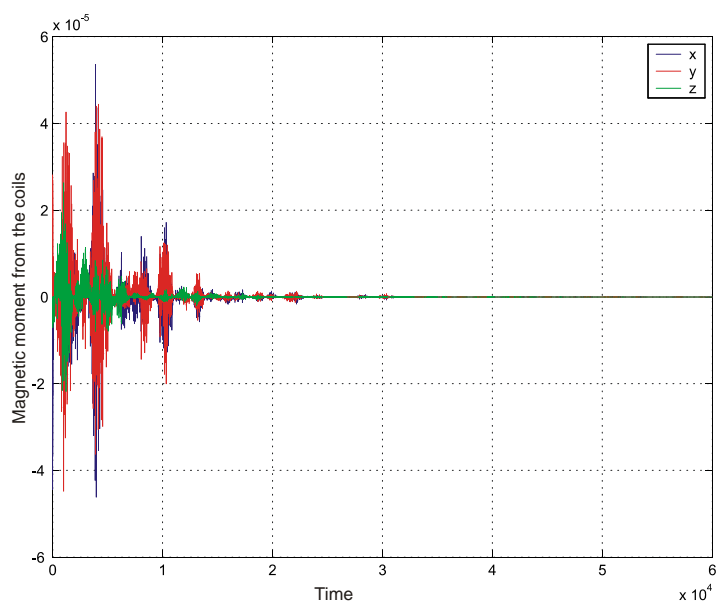

Fig. 6. The magnetic moment from the three coils
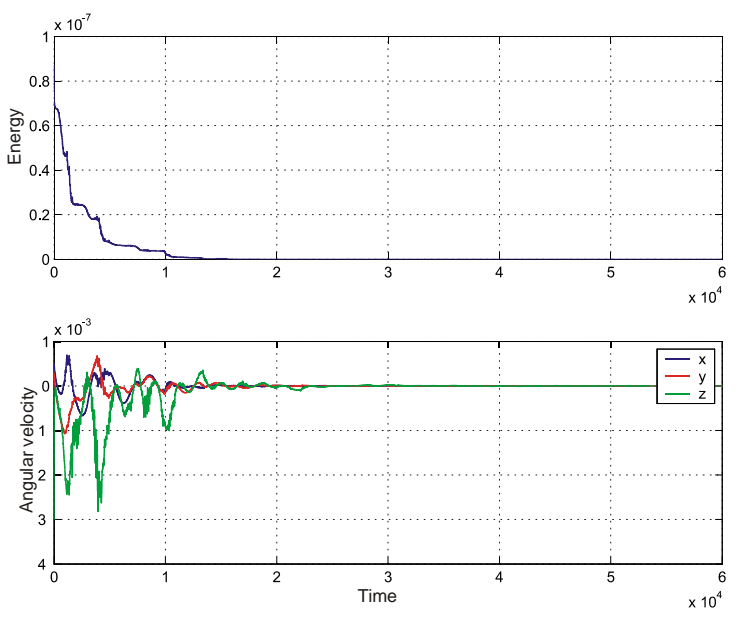

Fig. 7. Energy and angular velocity coils are used as actuators and the stability result is established by using Matrosovs theorem. The theoretical results have been confirmed by simulations on a model of the picosatellite nCube.

\section{ACKNOWLEDGEMENTS}

The former Dept. of Engineering Cybernetics students B. Busterud, K.M. Fauske, K. Svartveit, F. Indergaard and E.J. Øverby have all contributed to the design of the ADCS for nCube.

\section{REFERENCES}

[1] R. Wisniewski and M. Blanke, "Fully magnetic attitude control for spacecraft subject to gravity gradient," Automatica, vol. 35, pp. 1201$1214,1999$.

[2] "Http://www.rocketrange.no/ncube/," web site of the Ncube project, March 1st, 2004.

[3] A.-R. Riise, B. Samuelsen, N. Sokolova, H. Cederblad, J. Fasseland, C. Nordin, J. Otterstad, K. Fauske, O. Eriksen, F. Indergaard, K. Svartveit, P. Furebotten, E. Sæther, and E. Eide, "Ncube: The first norwegian student satellite," in In Proceedings of The17th AIAA/USU Conference on Small Satellites, 2003.

[4] J. Gravdahl, E. Eide, A.Skavhaug, K. Svartveit, K. M. Fauske, and F. M. Indergaard, "Three axis attitude determination and control system for a picosatellite: Design and implementation,", in Proceedings of the 54th International Astronautical Congress. Bremen, Germany: IAF, 2003.

[5] O. Egeland and J. Gravdahl, Modeling and Simulation for Automatic Control. Trondheim, Norway: Marine Cybernetics, 2002.

[6] J. Wertz, Space Mission Analysis and Design, 3rd ed. London: Kluwer academic publishers, 1999.

[7] R. Kristiansen, "Attitude control of mini satellite," Master's thesis, NTNU, 2000.

[8] P. Hughes, Spacecraft Attitude Dynamics. New York: Wiley, 1986.

[9] P. Soglo, "3-aksestyring av gravitasjonsstabilisert satelitt ved bruk av magnetspoler," Master's thesis, NTNU, 1994.

[10] R. Wisniewski, "Satellite attitude control using only elecromagnetic actuation," Ph.D. dissertation, Dept. of Control Engineering, Aalborg University, Denmark, 1996.

[11] O. Egeland, "Norwegian ionospheric small satellite experiment attitude control and determination system," Sintef, Tech. Rep. 94-72-W, 1994

[12] C. Damaren, "Comments on "fully magnetic attitude control for spacecraft subject to gravity gradient"," Automatica, vol. 38, p. 2189, 2002.

[13] M. Vidyasagar, Nonlinear Systems Analysis. Englewood Cliffs, NJ: Prentice-Hall, 1993.

[14] V. Matrosov, "On the stability of motion," J. Appl. Math. Mech, vol. 26, pp. 1337-1353, 1962.

[15] A. Loria, E. Panteley, D. Popovic, and A. Teel, "An extension of matrosov's theorem with application to stabilization of nonholonomic control systems," in Proccedings of the 41st IEE Conference on Decision and Control, Las Vegas, NV, 2002, pp. 1528-1533. 